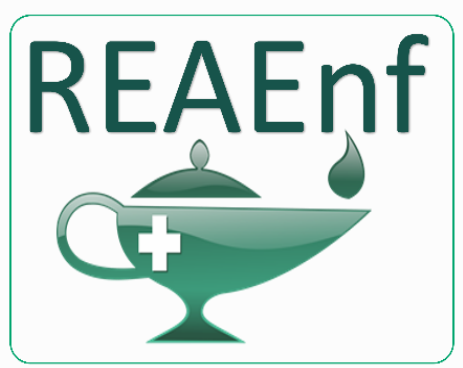

Revista Eletrônica Acervo Enfermagem
REVISÃO BIBLIOGRÁFICA

Recebido em: 9/2020

Aceito em: 10/2020

Publicado em: 11/2020

\title{
A prática de enfermagem frente aos pacientes portadores de esquizofrenia
}

\author{
Nursing practice in front of patients with schizophrenia \\ Práctica de enfermería frente a pacientes con esquizofrenia
}

Adinea Oliveira de Jesus Cardoso ${ }^{1 *}$, Gilseane Torres de Carvalho ${ }^{1}$, Tainara Santos de Matos ${ }^{1}$.

\begin{abstract}
Resumo: Esse artigo tem como objetivo geral compreender a prática de enfermagem frente aos pacientes portadores de esquizofrenia, considerando que o cuidado humanizado do enfermeiro junto a esse paciente é de extrema importância, pois visa a uma assistência holística estabelecendo vínculos do paciente e seus familiares. Ao desenvolver os cuidados é fundamental que o profissional reconheça os fatores relevantes para desenvolvê-lo e atentar-se a necessidade de ajudar o paciente e sua família a conhecer melhor a doença. No que se refere à metodologia, o estudo foi constituído de uma pesquisa descritiva e exploratória no intuito de se familiarizar mais profundamente com o fenômeno investigado. A pesquisa bibliográfica é um levantamento mais abrangente de referência bibliográfica sobre o tema escolhido. A enfermagem tem que desenvolver-se na área psiquiátrica e investir em pesquisa visto que tem pouco a trabalhos científicos publicados sobre o tema melhorando assim a assistência prestada, pois a mesma será baseada em conhecimento específico de Enfermagem. De forma geral pode-se concluir que estudos sobre doenças mentais, em especial, a Esquizofrenia, precisam ainda de aprofundamento e esclarecimento, para que com o tempo se possa entendê-la melhor e com isso identificar até mesmo formas de evitar seu desencadeamento.
\end{abstract}

Palavras-chave: Assistência de enfermagem, Esquizofrenia, Paciente.

\begin{abstract}
This article has as general objective to understand the nursing practice in the face of patients with schizophrenia, considering that the humanized care of nurses with this patient is extremely important, because it aims at holistic care establishing bonds between the patient and his/her family members. When developing care, it is essential that the professional recognizes the relevant factors to develop it and to pay attention to the need to help the patient and his/her family to know the disease better. With regard to the methodology, the study consisted of a descriptive and exploratory research in order to become more familiar with the investigated phenomenon. Bibliographic research is a more comprehensive survey of bibliographic reference on the chosen theme. Nursing has to develop in the psychiatric area and invest in research since it has little scientific work published on the theme thus improving the care provided, because it will be based on specific knowledge of Nursing. In general, it can be concluded that studies on mental diseases, especially schizophrenia, still need deepening and clarification, so that over time it can be better understood and thus identify even ways to avoid its triggering.
\end{abstract}

Keywords: Nursing care, Schizophrenia, Patient.

\footnotetext{
1 União Metropolitana Unime Feira de Santana LTDA. Feira de Santana - BA.

*E-mail: gilseane_@hotmail.com
} 
Resumen: Este artículo tiene como objetivo general entender la práctica de enfermería frente a pacientes con esquizofrenia, teniendo en cuenta que la atención humanizada de las enfermeras con este paciente es extremadamente importante, ya que tiene como objetivo la atención integral establecer lazos entre el paciente y sus familiares Al desarrollar la atención, es esencial que el profesional reconozca los factores relevantes para desarrollarla y preste atención a la necesidad de ayudar al paciente y a su familia a conocer mejor la enfermedad. Con respecto a la metodología, el estudio consistió en una investigación descriptiva y exploratoria con el fin de familiarizarse con el fenómeno investigado. La investigación bibliográfica es una encuesta más completa de referencia bibliográfica sobre el tema elegido. Enfermería tiene que desarrollarse en el área psiquiátrica e invertir en investigación ya que tiene poco trabajo científico publicado sobre el tema mejorando así la atención prestada, porque se basará en conocimientos específicos de enfermería. En general, se puede concluir que los estudios sobre enfermedades mentales, especialmente la esquizofrenia, todavía necesitan profundizar y aclarar, para que con el tiempo pueda entenderse mejor y así identificar incluso formas de evitar su desencadenamiento.

Palabras clave: Cuidados de enfermería, Esquizofrenia, Paciente.

\section{INTRODUÇÃO}

A esquizofrenia é psicopatologia crônica caracterizada por alterações persecutórias como delírios, alucinações, alterações de fala, comportamento, agitação e irritabilidade, embotamento afetivo, catatonismo e isolamento social. Essa psicopatologia é considerada um problema de saúde pública devido à incapacitação do individuo causando um impacto, na maioria das vezes, devastador no seu convívio social, familiar e econômico (GIRALDI A e CAMPOLIM S, 2014).

É uma doença que afeta a zona do eu alterando a capacidade do individuo de convivência com a realidade. A sua sintomatologia é dividida em sintomas positivos: aqueles que possuem melhores prognósticos e os sintomas negativos: característicos da doença em sua forma crônica (ARAÚJO AC e NETO FL, 2014). No que se refere à incidência, em geral, os homens têm um início em torno dos 18-25 anos apresentando sintomas negativos, tendência à solidão, probabilidade maior de permanecer solteiro devido o início precoce, pior resposta ao tratamento e alterações cerebrais estruturais. E as mulheres em torno dos 25-35 anos. Uma das hipóteses para essa diferença é que as mulheres possuam um fator de proteção: o estrógeno e geralmente elas possuem ocupação como trabalho, filhos, casamento ou são separadas (CORDEIRO FR, et al., 2012).

A esquizofrenia compromete a vida do paciente devido seus delírios e sua vida estressante aumentando assim o risco de suicídio devendo assim o tratamento ser planejado e implementado pela equipe de saúde. Por ser de causa multifatorial seja ela genética, bioquímica, fisiológica ou estresse psicológico a psicopatologia em questão resulta num enigma devido o sofrimento excessivo que o familiar, sociedade e o próprio indivíduo passam já que a mesma não possui cura levando a uma deteriorização ocupacional, social e afetiva (TAN SC, et al., 2012).

Considerando que a esquizofrenia não é sinônimo de violência, este estudo justificou-se, pois, conhecer os problemas que o portador de esquizofrenia enfrenta no seu dia-a-dia, entender como o enfermeiro vai atuar junto a esse paciente e sua família e mostrar para a sociedade que desde que o portador da esquizofrenia seja tratado e bem cuidado que ele pode conviver no meio social de forma satisfatória a fim de que possa promover a reinserção social como a familiar (TAVARES CMM, et al., 2012).

Sendo assim, o presente estudo delimita-se a responder a seguinte problemática de pesquisa: quais os aspectos importantes na assistência de enfermagem ao portador de esquizofrenia? A fim de responder a problemática exposta delimitou-se como objetivo geral compreender a prática de enfermagem frente aos pacientes portadores de esquizofrenia. Ademais, a relevância do estudo é ampliar o conhecimento dos profissionais de enfermagem que exercem a prática da Saúde Mental para que prestem um cuidado sistematizado aos seus pacientes. 


\section{REVISÃO BIBLIOGRÁFICA}

$\mathrm{Na}$ antiguidade, os pacientes afetados pela doença mental, mais conhecida como "esquizofrenia" tinham curso deteriorante em longo prazo e sintomas clínicos comuns de alucinações e delírios. Kraepelin E e Diefendorf AR, 1966 denominaram a esquizofrenia de Demência Precoce. Esses critérios baseavam-se em três características principais sintomatologia, etiologia e evolução.

O critério sintomatológico constituía-se, na descrição dos sintomas observados, tais como déficits da atenção e da compreensão, as alucinações, principalmente auditivas, o pensamento sonoro, a vivência de influência, o embotamento da afetividade e as mudanças do comportamento. $O$ critério etiológico pressupunha a existência de um quadro endógeno. O critério evolutivo caracterizava-se pela evolução desfavorável e pela invalidez psíquica. Daí a denominação de Demência Precoce (GIRALDI A e CAMPOLIM S, 2014).

Devido à denominação de o estigma que o portador de esquizofrenia sofre é constante mesmo nos dias atuais (KRAEPELIN E e DIEFENDORF AR, 1966). A maioria dos familiares e profissionais tem a percepção que o paciente estará destinado a ser improdutivo socialmente. A esquizofrenia é uma psicopatologia caracterizada por distúrbios de pensamentos, alucinações e delírios. Causa incapacidade da vida socioeconômica do portador devido ao difícil diagnóstico e intervenções no primeiro episódio. É altamente prevalente, mas com baixa incidência (TAN SC, et al., 2012).

Desse modo, observa-se que o curso da doença é altamente incapacitante alterando a qualidade de vida do portador, pois a mesma causa disfunções ocupacionais, sociais, pessoais e familiares e afetando, possivelmente, a qualidade de vida tanto dos pacientes quanto dos seus familiares (CORDEIRO FR, et al., 2012).

A esquizofrenia se inicia geralmente no fim da adolescência ou no inicio da fase adulta. A sua prevalência na população geral é de $1 \%$. O individuo começa a apresentar alterações de comportamento social, sexual e de personalidade e convívio. Esse pré-mórbido é fator indicativo para o desenvolvimento da esquizofrenia, este está dividido em quatro fases nas ideias de (CORDEIRO FR, et al., 2012). A primeira se refere à personalidade esquizóide, na qual o paciente começa a apresentar as primeiras sintomatologias, a esquizofrenia propriamente dita onde os sintomas são mais evidentes e, por fim, a fase residual, onde os sintomas são mais explícitos.

Desse modo, observa-se que o curso da doença é altamente incapacitante alterando a qualidade de vida do portador, pois a mesma causa disfunções ocupacionais, sociais, pessoais e familiares e afetando, possivelmente, a qualidade de vida tanto dos pacientes quanto dos seus familiares (CORDEIRO FR, et al., 2012). A etiologia da esquizofrenia é altamente complexa. Acredita-se que vários fatores influenciam no aparecimento da doença, tais como: biológicos, psicológicos, ambientais, sociais e genéticos. A hipótese diátese-estresse diz que o individuo pode ter uma predisposição à patologia e devido a um estresse biológico ou ambiental ela se desenvolve (CARVALHO JC, 2012).

Indivíduos que possuem um parentesco de primeiro grau portador de esquizofrenia têm um risco dez vezes maior que a população em geral. A concordância é maior em gêmeos monozigóticos do que em gêmeos dizigóticos, mas o fator ambiental é extremamente importante para o desenvolvimento da mesma (OLIVEIRA R e FUREGATO ARF, 2012).

Há também uma relação entre a exposição do feto a infecção viral observou-se que era mais intensa quando esta ocorria no período do segundo trimestre de gestação, período considerado crítico, pois está em formação o sistema límbico e cortical. O estresse psicossocial é um fator importante, pois ele, na maioria das vezes, desencadeia o surto do paciente (GIRALDI A e CAMPOLIM S, 2014).

Os fatores biológicos atribuem a um distúrbio entre as ligações de neurotransmissores cerebrais. A hipótese da Dopamina é sugestiva que o excesso da atividade neuronal dependente de dopamina produz e libera maior quantidade desse neurotransmissor nos terminais nervosos, a maior sensibilidade dos receptores e menor atividade dos antagonistas (SARIAH AE, et al., 2014). 
Outra hipótese é que haja alguma anormalidade entre os neurotransmissores norepinefrina, serotonina, acetilcolina e ácido gama-aminobutírico e nos neurorreguladores como prostaglandinas e endorfinas. Sugere-se que o corpo produz substâncias que toma o lugar das vias neurotransmissoras habituais no cérebro (ELOIA SC, et al., 2014).

As influências fisiológicas algumas possuem importâncias etiológicas mesmo que seu mecanismo específico não foi esclarecido são eles: a hipótese das infecções viróticas, anormalidades anatômicas, alterações histológicas e condição física. As influências psicológicas são relevantes, mas difícil de prová-las na prática. As teorias psicanalíticas e familiares tentam explicar que a visão com a realidade foi deturpada devido a mecanismos próprios que as crianças utilizaram para se livrar das demandas que o mesmo não aceite (ARAÚJO AC e NETO FL, 2014).

A realização de exames laboratoriais como: hemograma, dosagens de eletrólitos, glicose, lipídios sanguíneos, teste a função hepática, renal e tireoidiana, sorologia para HIV, sífilis e hepatite e triagem taxológica na urina são realizados para dar um diagnóstico fidedigno evitando uma avaliação errônea devido ao uso de drogas e distúrbios metabólicos. Também é realizado Eletroencefalograma, tomografia computadorizada (TC) e ressonância magnética (RM) exames para diferenciar entre quadros orgânicos e a psicopatologia (GOMES MS e MELLO R, 2012).

A intervenção pertinente depende do acompanhamento farmacológico, psicossocial e inclusão familiar. $O$ diagnóstico e a delimitação do tratamento devem ser individuais visto que cada paciente possui características próprias da patologia. A assistência de enfermagem deve ter como foco principal a saúde e a qualidade de vida da pessoa, família e coletividade. Nesse contexto, os profissionais de enfermagem devem atuar conjuntamente de modo a promover a saúde em seus diversos aspectos (GIRALDI A e CAMPOLIM S, 2014).

O Profissional de Enfermagem exerce suas atividades com competência para a promoção do ser humano na sua integralidade, de acordo com os princípios da ética e da bioética. A enfermagem psiquiátrica é principalmente composta de relacionamentos interpessoais seja ele com o paciente ou seus familiares no qual se observa aspectos biopsicossociais do ser humano (CORDEIRO FR, et al., 2012).

No aspecto biológico a enfermagem atua no controle das medicações e acompanha a saúde em seus diversos sistemas do paciente e da família. Já no campo psicossocial ela está inserida em atividades ocupacionais, visita domiciliar e o contato direto com o paciente que possibilita o diálogo adquirindo confiança do mesmo e tecendo uma relação enfermeiro-paciente sadia o qual possibilita a orientação do mesmo sobre sua patologia e principalmente como seguir o tratamento (CARDOSO L e GALERA SA, 2012).

Os enfermeiros têm maiores dificuldades em trabalhar com pacientes na fase aguda, pois despertam nele sentimentos de impotência pela demanda sintomática e pela incerteza prognóstica e tem maior satisfação em trabalhar com os crônicos devido o trabalho reinserção social (CORDEIRO FR, et al., 2012). Devido ao contato direto com paciente psiquiátrico o Enfermeiro e a equipe de enfermagem fica submetida ao stress continuo levando a insatisfação para com seu trabalho devido ao tempo prolongado. Embora o trabalho seja exaustivo quando o resultado é favorável os profissionais se sentem gratificados por participar da evolução do seu paciente (OLIVEIRA R e FUREGATO ARF, 2012).

$\mathrm{Na}$ prática, observa-se que enfermeiros psiquiátricos, com frequência, encontram dificuldade em implementar os cuidados de enfermagem aos esquizofrênicos, devido às características dessa doença mental. As manifestações tipo alucinações, delírios, autoagressão e agitação psicomotora provocam ansiedade nos enfermeiros, dificultando o estabelecimento da comunicação interpessoal e a concretização da relação de ajuda (GIRALDI A e CAMPOLIM S, 2014).

A comunicação é muito importante para o enfermeiro, pois através dela o enfermeiro desperta o interesse do esquizofrênico pela vida digna e de participação no seu meio familiar e social, valorizando e incentivando os mesmos a aderir e participar do seu tratamento. Cabe a enfermagem a buscar e estimular no paciente sua autonomia, pois a mesma é uma conduta ética no processo de cuidar (CARDOSO $L \mathrm{e}$ GALERA SA, 2012). 
A enfermagem como integrante da equipe multidisciplinar deve estar apta a desenvolver suas atividades referentes ao cuidado sistematizado ao portador, mas isso não é o que ocorre frequentemente, pois os mesmos relatam insegurança com pacientes agressivos que estão na fase aguda da doença, falta de tempo e sobrecarga de trabalho como impasse para realizar uma assistência correta (TAN SC, et al., 2012).

O enfermeiro como profissional deve capacitar-se para sentir segurança na sua atuação profissional procurando além da graduação um diferencial que o permita ser preparados para atuar com os portadores de esquizofrenia identificando sinais, sintomas, remissão, tratamento efetivo, a não adesão ao tratamento e executar suas intervenções embasados no conhecimento cientifico adquirido juntamente a sua prática profissional (CORDEIRO FR, et al., 2012).

A enfermagem deve estar atenta aos sinais que podem surgir ao decorrer do tratamento como a obesidade, hiperlipidemia, hiperprolactinemia, disfunção sexual, problemas cardiovasculares, leucopenia benigna e agranulacitose, alergias, alterações das enzimas hepáticas, retenção ou incontinência urinária (CORDEIRO FR, et al., 2012).

A SAE (sistematização da assistência de enfermagem) organiza o protocolo de cuidar e melhora a qualidade da assistência prestada e sem dúvidas deve ser utilizado na área psiquiátrica desde as alterações psicopatológicas a de saúde como um todo. Os diagnósticos de enfermagem possibilitam ao profissional enfermeiro autonomia e autodeterminação, para isso utiliza-se a taxonomia da NANDA (CARDOSO L e GALERA SA, 2012).

O diagnóstico de enfermagem foi definido na $9^{a}$ conferência da NANDA como "um julgamento clínico das respostas do individuo, da família ou da comunidade aos processos vitais ou aos problemas de saúde atuais ou potenciais, os quais o enfermeiro é responsável" (CARDOSO L e GALERA SA, 2012). É necessário que se oriente o paciente quanto ao uso dos medicamentos e seus efeitos colaterais, verificando-se o uso correto dos medicamentos, programar avaliações biopsicossociais com atenção às características culturais do cliente, criando e implementando projetos para melhorar as condições de saúde do paciente e de seus familiares, aconselhar paciente e familiares sobre aspectos da doença, do tratamento e recursos disponíveis (CORDEIRO FR, et al., 2012).

Deve-se dar uma atenção holística aos pacientes com transtornos mentais desde a admissão até sua alta hospitalar, acompanhando sua evolução diariamente através do monitoramento de todos os seus sinais vitais e observação dos sinais e sintomas. É de fundamental importância que os profissionais de enfermagem tenham conhecimento técnico-científico na identificação da Esquizofrenia em pacientes hospitalizados, tendo uma assistência de enfermagem sistematizada, visando um cuidado qualificado a estes pacientes, fazendo as intervenções cabíveis para que eles tenham uma melhor qualidade de vida (TAN SC, et al., 2012).

Cabe ao profissional de enfermagem um papel de grande relevância: o de estimular e facilitar para o paciente e seus familiares, não abandonem o tratamento. Que se comprometam em auxiliar nas dificuldades apresentadas e que estejam dispostos a dar tudo de si ao assumirem tarefas relacionadas ao referido tema. O fortalecimento dos vínculos e a confiança serão fatores importantíssimos para o equilíbrio e plena reabilitação do paciente. $O$ enfermeiro poderá auxiliar significativamente no enfrentamento das dificuldades e limitações vivenciadas pelo paciente. Por meio da autoconfiança o indivíduo poderá fortificarse para sua plena reinserção na sociedade (ARAÚJO AC e NETO FL, 2014).

Os cuidados e intervenções de enfermagem psiquiátrica são essenciais para proporcionar uma melhor qualidade de atendimento aos pacientes e condições adequadas para tratamento. Portanto deve haver um planejamento de todos os processos desde a fase de identificação ate o processo de acompanhamento e efetivação de cuidados (OLIVEIRA R e FUREGATO ARF, 2012).

Observa-se que a falta de profissionais de enfermagem treinados e capacitados é uma das grandes barreiras a serem vencidas quando se fala de cuidados em psiquiatria. O comportamento do cliente pode parecer extravagante e ilógico às pessoas que cuidam dele, mas e necessário observar e analisar profundamente o que o cliente esta querendo expressar (CARDOSO L e GALERA SA, 2012). 
O enfermeiro tem um papel decisivo e importante na recuperação do cliente. A meta ao cuidar do paciente com esquizofrenia é mantê-lo em uma realidade coerente o maior período de tempo possível, utilizando adequadamente todas as estratégias necessárias, para que ele possa ser reintegrado a sociedade (CORDEIRO FR, et al., 2012).

Sendo assim, é inegável que o profissional de enfermagem é definitivamente fundamental no desenvolvimento e tratamento de pacientes com o transtorno esquizofrênico, e também em qualquer outro transtorno mental, e que estas por sua vez devem dispor-lhes de todo o cuidado que estiver ao seu alcance.

\section{CONSIDERAÇÕES FINAIS}

A assistência de enfermagem será direcionada aos pacientes de forma sistematizada e correta somente quando os profissionais atuantes se especializarem colocando em prática o conhecimento adquirido. Percebe- se que os profissionais de enfermagem negligenciam a sua atividade devido à característica da patologia e a falta de conhecimento específico para manter a comunicação e os cuidados rotineiros. A maioria deles se sente impotentes ao tentar dominar as complicações que fogem ao seu controle como o suicídio, fuga e autoagressão. Diante disso a necessidade de aprimoramento profissional é vista como indispensável, pois os mesmos sentem a necessidade de saber como lidar com um paciente tão peculiar como o esquizofrênico aprendendo técnicas de abordagem e aplicando a cada individuo de forma única já que cada paciente possui suas diferenças e necessidades particulares. De forma geral pode-se concluir que estudos sobre doenças mentais, em especial, a Esquizofrenia, precisam ainda de aprofundamento.

\section{REFERÊNCIAS}

1. ARAÚJO AC, NETO FL. A Nova Classificação Americana Para os Transtornos Mentais. - o DSM-5. Revista brasileira de Terapia comportamental e Cognitiva, 16(1), 67-82, 2014.

2. CARDOSO L, GALERA SA. O cuidador e a sobrecarga do cuidado à saúde de pacientes egressos de internação psiquiátrica. Acta paul. enferm. 2012.

3. CARVALHO JC. Diagnósticos e intervenções de enfermagem centradas no processo familiar da pessoa com esquizofrenia. Revista Portuguesa de Enfermagem de Saúde Mental. Porto, 8, 557, 2012.

4. CORDEIRO FR, et al. Cuidados de Enfermagem à pessoa com esquizofrenia: Revisão Integrativa. Revista de Enfermagem UFSM. Santa Maria, 2(1), 174-181, 2012.

5. ELOIA SC, et al. Sobrecarga do cuidador familiar de pessoas com transtorno mental: Uma revisão integrativa. Revista Saúde em Debate, Rio de Janeiro, 38(103), 996-1007, 2014.

6. FONSECA LM, GALERA SAF. Expressões utilizadas por familiares ao relatarem experiências de conviver com o adoecimento mental. Acta Paulista de Enfermagem. São Paulo, 25(1), 61-67, 2012.

7. GIACON BCC, GALERA SAF. Ajustamento familiar após o surgimento da esquizofrenia. Revista Brasileira Enfermagem, Brasília, v. 66, n. 3. 2013.

8. GIRALDI A, CAMPOLIM S. Novas abordagens para esquizofrenia. Cienc. Cult. São Paulo, 66(2), 6-8, 2014.

9. GOMES MS, MELLO R. Sobrecarga gerada pelo convívio com o portador de esquizofrenia: a enfermagem construindo o cuidado à família. Revista Eletrônica Saúde Mental Álcool e Drogas. São Paulo, 8(1), 2-8, 2012.

1. 10 KRAEPELIN E, DIEFENDORF AR. Clinical psychiatry: Nova York: Macmillan Company, 1966.

10. OLIVEIRA R, FUREGATO ARF. Um casal de idosos e sua longa convivência com quatro filhos esquizofrênicos. Rev. Esc. Enferm. USP, 2012.

11. SARIAH AE, et al. Risk and protective factors for relapse among individuals with Schizophrenia: A Qualitative Study in Dar es Salaam, Tanzania. BMC Psychiatry. 240(14), 1-12, 2014.

12. SOUZA J, et al. Estratégia de Saúde da Família: Recursos Comunitários na Atenção à Saúde Mental. Acta Paulista de Enfermagem, 26(6), 2013, 594-600.

13. TAN SC, et al. Burden and coping strategies experienced by caregivers of persons with schizophrenia in the community. Journal of Clinical Nurse. 2012.

14. TAVARES CMM, et al. Atenção de enfermagem à família do portador de transtorno mental: Contribuições para a educação permanente. Ciência, Cuidado e Saúde, Maringá, 11(4), 767-774, 2012. 